\title{
The experimental study of increasing the efficiency of emulsion separation
}

\author{
Vadim Zinurov ${ }^{1}$, Ilnar Sharipov ${ }^{1}$, Oksana Dmitrieva ${ }^{2,}$, and Ilnur Madyshev ${ }^{2}$ \\ ${ }^{1}$ Kazan State Power Engineering University, 420066, Krasnoselskaya Street, 51, Kazan, Russia \\ ${ }^{2}$ Kazan National Research Technological University, 420015, Karl Marx Street, 68, Kazan, Russia
}

\begin{abstract}
The aim of this research paper is to compare the operation efficiency of two types of coalescents: insert, made of high porous material and flat baffles. For this purpose, the method of physical experiment was applied. This research paper shows that the use of them in the settling tank allows to increase the efficiency and velocity of water-oil emulsion separation with an increase of oil concentration in the original mixture from 15 up to $25 \%$. The experimental studies also show that the most effective coalescers are the baffles, than the inserts, made of highly porous cellular material, due to the fact that the cells are quickly clogged with heavy oil components, which leads to a more complex flow structure through them, therefore, the process of mixing oil and water compounds is intensified and prevails over the coalescence process. The velocity of oilwater emulsion separation when using the inserts, made of highly porous cellular material, and baffles in comparison with the settling tank without inserts, increases on average by 10.9 and $14.5 \%$.
\end{abstract}

\section{Introduction}

Due to the high water encroachment of oil fields, the significant capital costs for the objects of collection and transportation system (products from the oil field wells) increase. As a result, most of oil fields are beyond the profitability, cost-effectiveness and development of them stops, while the infrastructure becomes unclaimed. Therefore, to increase the efficiency of water-oil emulsions stratification at the oil production fields is a very urgent and relevant task. The importance of this task is also high from an ecological point of view - in most cases, the extracted water from emulsions is pumped back into the aquifer, which is an economical way to dispose the large volumes of stratal water, but the quality of this water shall meet the criteria in order to prevent damage to the stratum when pumping back [1-4].

The most common ways to separate the water-oil emulsions are mechanical, chemical, thermal, and electrical. The simplest from technical point of view and cheapest method is the mechanical one, which includes settling, centrifugation and filtration, which allows it to be implemented and used at the oil fields with minimum capital and operating costs. The chemical, thermal, and electrical methods are the most effective, but at the same time, the

\footnotetext{
*Corresponding author: ja_deva@mail.ru
} 
complexity and cost of using those is high. In most cases, the oil refinery plants use them as the next stage of water-oil emulsions purification from water and various impurities after the mechanical purification stage. Thus, the most cost-effective method for separating the water-oil emulsions in this case is the mechanical one. In order to implement the mechanical method, the settling tanks, centrifuges and filters shall be used. The process of separating the water-oil emulsions is based on the physical properties of liquids and the force of gravity. The mechanical method is widely used with watered oil, but is not effective for the deep dehydration. It should be noted that year by year, the water encroachment of oil fields increases. Thus, increasing the efficiency of water-oil emulsions separation in mechanical apparatuses is a very urgent and relevant task [5-9].

As noted above, the apparatuses that operate according to the mechanical method are divided into several types: settling tanks, centrifuges and filters. Among them, the main equipment at the oil fields are the settling tanks, since they do not need additional devices, for example, those, providing heating of water-oil emulsions. The use of centrifuges and filters at the oil fields is complicated by the need for additional maintenance and economic costs. High energy consumption is required to create high-speed centrifugal accelerations within the centrifuges. The use of various filter models is accompanied by the need for periodical replacement of consumables or cleaning of them. Therefore, the most promising way to increase the efficiency of water-oil emulsions separation at the oil fields is to study the various methods of improvement of settling tanks. One of these methods is the study of various inserts, to be installed inside of settling tanks, which have the form of webs, shields and other geometric bodies that increase the velocity of water-oil emulsions stratification into components by changing the flow structure when skirting the obstacles. It should be noted that nowadays the flow rate of water-oil emulsions in settling tanks does not exceed few millimeters per second, since at higher velocities the efficiency of emulsions separation significantly decreases due to the mixing of phases [10-20].

The purpose of this research paper is to perform experimental study of various inserts in the settling tank for the possibility of increasing the efficiency and velocity of water-oil emulsions separation.

\section{Objects under the study and research methods}

The authors of this research paper developed two different models of experimental inserts: baffles and inserts, made of highly porous (void) cellular material (Figure 1). The baffles were the rectangular plates of $60 \times 60 \times 20 \mathrm{~mm}$ (length $\times$ height $\times$ depth), within which the rectangular holes were executed with a step of $10 \mathrm{~mm}$. The inserts, made of highly porous cellular material, had the following geometric dimensions: length $-60 \mathrm{~mm}$, height -60 $\mathrm{mm}$, depth $-20 \mathrm{~mm}$ and cell diameter $-5 \mathrm{~mm}$. The porosity of cellular material medium was 0.7 . The authors made an assumption that the developed inserts will allow to separate the water-oil emulsions in settling tanks with higher velocity and efficiency in comparison with the settling tank without any inserts. 


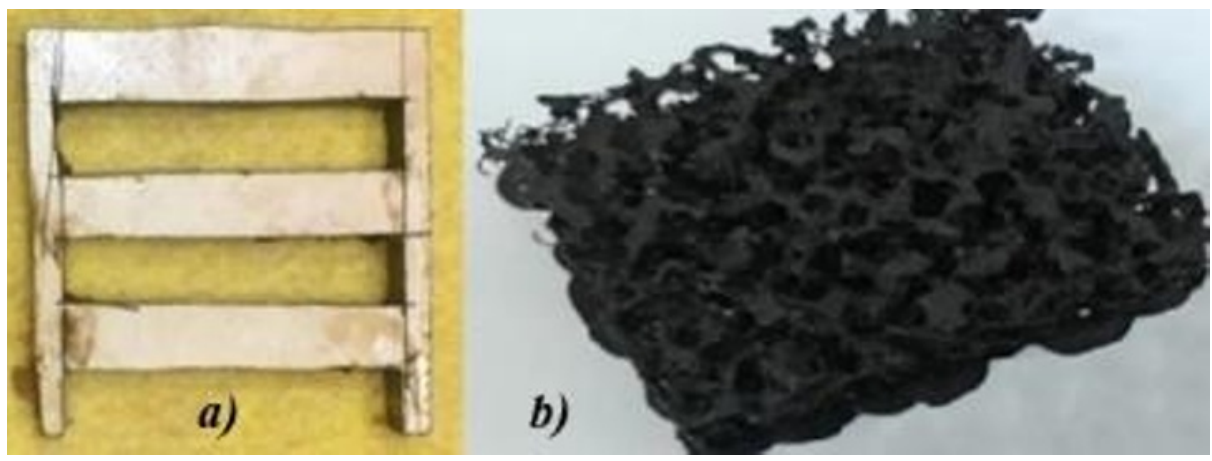

Fig. 1. Experimental models of inserts: $a$ ) baffle; $b$ ) insert, made of highly porous (void) cellular material.

In order to perform the experimental studies a laboratory apparatus (unit) was assembled, including rectangular settling tank, several modifications of inserts (baffles and inserts, made of highly porous cellular material) and auxiliary equipment for mixing water and oil, as well as studying the products of water-oil emulsion separation: control valves, samplers, flow meters, tank for re-mixing of separated emulsion and pump. The experimental studies can be summarized as follows: first, the treated water-oil emulsion was fed into the settling tank, where it was stratified into the components (water and oil), then the light phase (oil compounds) moved to the upper part of apparatus, the heavy phase (water) moved to the bottom part of apparatus. It should be noted that the control valves, designed to remove the light and heavy phases from the apparatus, were opened after the settling tank was completely filled with water-oil emulsion. In order to maintain the required flow rate of oil-water emulsion components through the control valves, the flow meters were used. After that, the samplers determined the mass fractions of oil and water in the light and heavy phases. Then, these two phases were fed to the re-mixing tank to form the oil-water emulsion, which was further fed to the settling tank by means of pump (Figure 2).

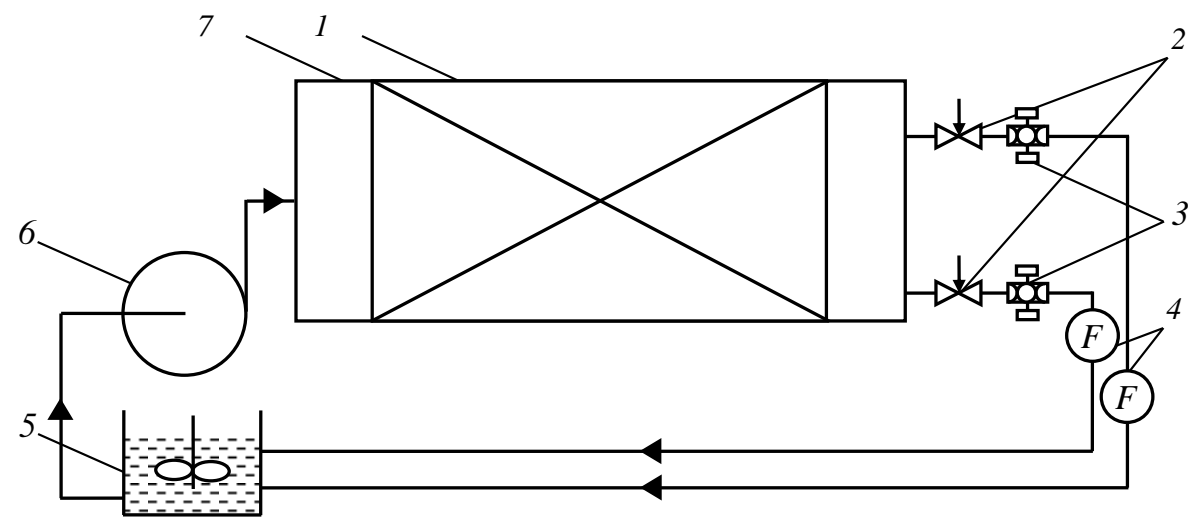

Fig. 2. Laboratory apparatus: 1 -insert under the study; 2 - control valves; 3 - samplers; 4 flowmeters; 5 - re-mixing tank; 6 - pump; 7 - settling tank.

In the course of experimental research the following parameters were assumed to be constant: ambient temperature $20^{\circ} \mathrm{C}$; water density $998.2 \mathrm{~kg} / \mathrm{m}^{3}$; coefficient of dynamic viscosity of water $0.001003 \mathrm{~kg} /(\mathrm{m} \cdot \mathrm{s})$; oil density $883 \mathrm{~kg} / \mathrm{m}^{3}$; coefficient of dynamic 
viscosity of oil $0.0198 \mathrm{~kg} /(\mathrm{m} \cdot \mathrm{s})$. In the course of study, the oil concentration in the original mixture varied from $15 \%$ up to $25 \%$.

The following formula was used to determine the efficiency of settling tank`s operation:

$$
E=x D-x F 1-x F,
$$

where $\overline{x_{D}}-$ mass fraction of oil in the light phase, $\mathrm{kg} / \mathrm{kg} ; \overline{x_{F}}-$ mass fraction of oil in the original mixture, $\mathrm{kg} / \mathrm{kg}$.

\section{Results and discussion}

The obtained results of experimental research are shown graphically in Figures 3-6. It should be noted that the graphs for the visual clarity show the intermediate lines of efficiency growth of water-oil emulsions separation at the appropriate speeds, so that we can see the most effective ranges of settling tank's operation when using the various inserts that were determined in the course of experiments. According to the obtained results, when the oil-water emulsion velocity in the settling tank increases, it leads to an increase in the efficiency of its separation into the constituent components as well. The efficiency of oilwater emulsion separation, with the oil concentration $C$ in the original mixture of $15-25 \%$, and an increase in the velocity from 0.047 up to $0.08 \mathrm{~m} / \mathrm{s}$, was on average equal to 63.1, 60.2 and $53.5 \%$ - when using the settling tank without inserts, with inserts, made of highly porous cellular material, and baffles, respectively. As can be seen, the average separation efficiency values were larger at higher speeds due to the fact that an increase in the velocity by $5-20 \%$ intensified the coalescence process. The developed inserts also acted as coalescers, i.e. they increased the number of merging drops of oil compounds, which were enlarged so that could be easily extracted from the water-oil emulsion, as a result of which the velocity of water-oil emulsion separation also increased. However, the more complex structure of emulsion flow, when using the inserts and due to their geometrical dimensions, intensified the process of mixing the oil and water phases, and, as a result, the efficiency of separating the water-oil emulsion in a settling tank without inserts was higher, but the separation process was slower. An increase of oil concentration in the original mixture led to an increase in the efficiency of separating the water-oil emulsion into the constituent components due to the fact that the number of oil compounds increased, therefore, the coalescence process was more intensive.

The efficiency of water-oil emulsion separation, with the oil concentration in the original mixture of $C=15 \%$, was on average equal to $50.9,47.4$, and $43.1 \%$ - when using the settling tank without inserts, with inserts, made of highly porous cellular material, and baffles, respectively. It should be noted that at the maximum velocity of water-oil emulsion separation, when using the baffles in the settling tank, equal to $0.066 \mathrm{~m} / \mathrm{s}$, the efficiency of its separation was $56.4 \%$, i.e. by $11.6 \%$ higher than at the maximum velocity, when using the inserts, made of high-porous cellular material, due to the fact that that the cells of highporous material were quickly clogged with oil components. Thus, the passage through it became more difficult, and it began to operate more as a mixer than a coalescer (Figure 3 ). 


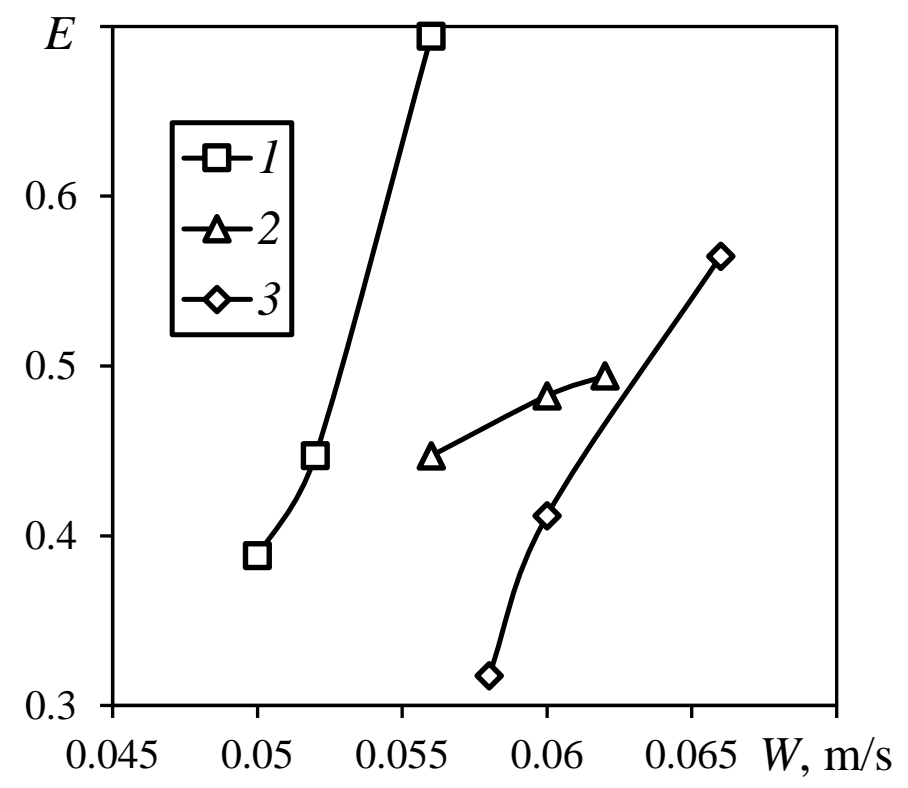

Fig. 3. The efficiency of separation on its velocity in the apparatus: 1 -without inserts; 2 -with inserts, made of highly porous cellular material; 3 - with baffles. $C=15 \%$.

The efficiency of water-oil emulsion separation, with the oil concentration in the original mixture of $20 \%$, was on average equal to $63.8,63.3$ and $55.1 \%$ - when using the settling tank without inserts, with inserts, made of highly porous cellular material, and baffles, respectively. With an increase of oil concentration in the original mixture from 15 up to $20 \%$, the efficiency of emulsion separation increased on average by $13.5 \%$ (Figure 4).

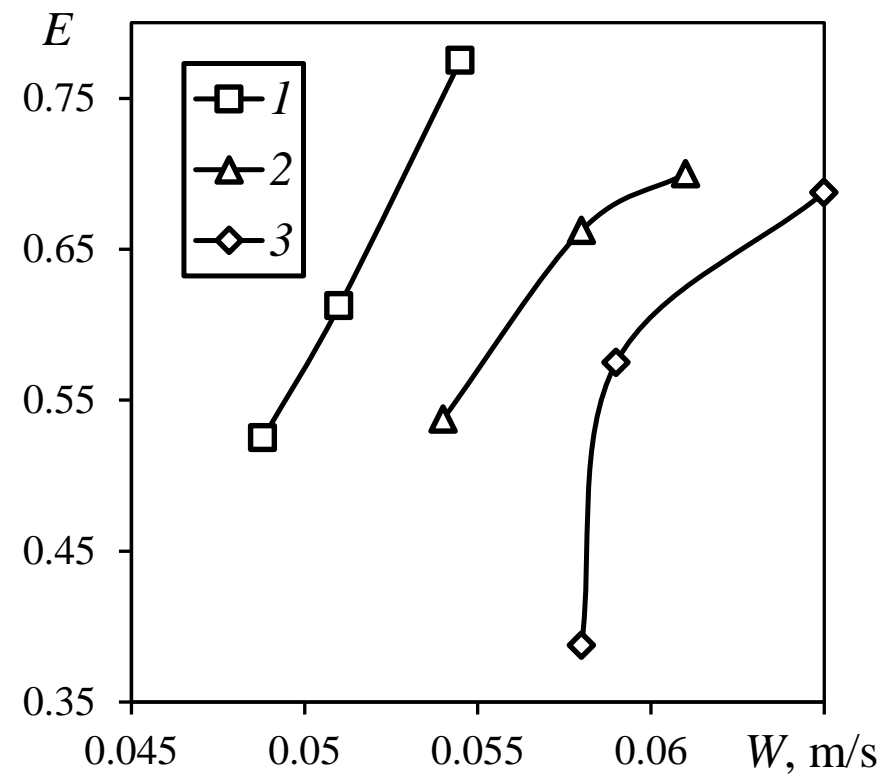

Fig. 4. The efficiency of separation on its velocity in the apparatus: 1 - without inserts; 2 - with inserts, made of highly porous cellular material; 3 - with baffles. $C=20 \%$. 
The efficiency of water-oil emulsion separation, with the oil concentration in the original mixture of $25 \%$, was on average equal to $74.6,69.8$ and $62.2 \%$ - when using the settling tank without inserts, with inserts, made of highly porous cellular material, and baffles, respectively. With an increase of oil concentration in the original mixture from 20 up to $25 \%$, the efficiency of emulsion separation into its constituent components was on average equal to $8.1 \%$ (Figure 5).

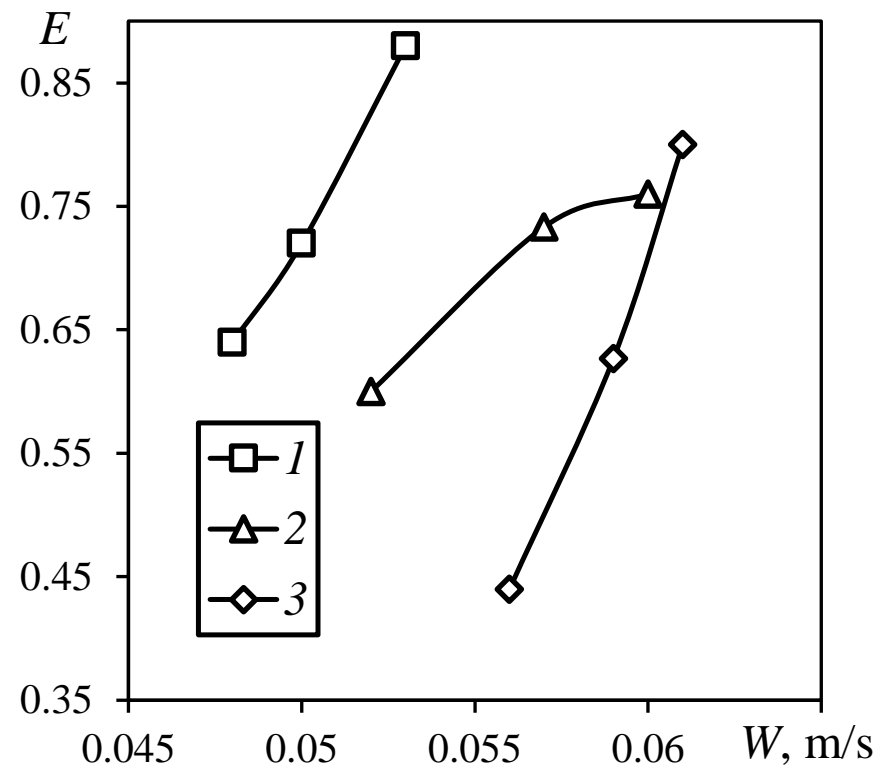

Fig. 5. The efficiency of separation on its velocity in the apparatus: 1 - without inserts; 2 -with inserts, made of highly porous cellular material; 3 - with baffles. $C=25 \%$.

In the course of all studies, it was found that the use of various inserts in the settling tank allowed to increase the velocity and efficiency of water-oil emulsions separation with an increase of oil concentration in the original mixture from 15 up to $25 \%$. The velocity of oil-water emulsion separation, when using the inserts, made of highly porous cellular material, and baffles, in comparison with the settling tank without inserts, increased on average by 10.9 and $14.5 \%$, respectively. The experimental studies show that the most effective coalescers are the baffles, than the inserts, made of highly porous cellular material, due to the fact that the cells are quickly clogged with heavy oil components, which leads to a more complex flow structure through them, therefore, the process of mixing oil and water compounds is intensified and prevails over the coalescence process.

\section{Conclusion}

Considering the fact that the settling tanks act as a first stage for the separation of oil-water emulsions, and one of the most important criteria in the oil-refining industry is the efficiency and velocity of this process, it follows that the use of inserts to intensify the separation of emulsions is a significant method, since they allow to increase the oil refining volumes during the crude oil production, therefore, economic profitability also increases. The advantages of these inserts are the ease of manufacture, the ease of use, low cost and maintainability.

The research was conducted with funding from the RF President`s grant project No. MK-616.2020.8. 


\section{References}

1. P. Zhang, G. Chen, et al., Sep. Purif. Technol., 210, 231-5 (2019) doi: 10.1016/j.seppur.2018.08.008

2. W. Jiang, Y. Chen, et al., Sep. Purif. Technol., 211, 259-68 (2019) doi: 10.1016/j.seppur.2018.09.069

3. S. Wang, W. Qin, and Y. Dai, Chin. J. Chem. Eng., 20, 239-45 (2012) doi: 10.1016/S1004-9541(12)60384-X

4. W. Chen, J. Peng, et al., Sep. Purif. Technol., 66, 591-7 (2009) doi: 10.1016/j.seppur.2009.01.009

5. M.T. Shah, H.B. Parmar, et al., J. Pet. Sci. Eng., 182, 106352 (2019) doi: 10.1016/j.petrol.2019.106352

6. I. Sadeghi, A. Aroujalian, et al., J. Membr. Sci., 430, 24-36 (2013) doi: 10.1016/j.memsci.2012.11.051

7. X. Wang, J. Yu, et al., Mater. Today, 19,403-14 (2016) doi: 10.1016/j.mattod.2015.11.010

8. Q. Zeng, Z. Wang Z, et al., J. Pet. Sci. Eng., 145, 83-94 (2016) doi: 10.1016/j.petrol.2016.03.015

9. A.V. Dmitriev, V.E. Zinurov, et al., E3S Web Conf., 110, 01026 (2019) doi: 10.1051/e3sconf/201911001026

10. M. Ochowiak, M. Matuszak, S., et al, J. Environ. Manage., 189, 22-8 (2017) doi: 10.1016/j.jenvman.2016.12.023

11. H. Gong, W. Li, et al., Sep. Purif. Technol., 238, 116397 (2020) doi: 10.1016/j.seppur.2019.116397

12. J.P. Veerapen, B.J. Lowry, and M.F. Couturier, Aquacultural Engineering, 33, 21-45 (2005) doi: 10.1016/j.aquaeng.2004.11.001

13. R. Tarpagkou, A. Pantokratoras, Powder Technol., 268, 139-49 (2014) doi: 10.1016/j.powtec.2014.08.030

14. O.S. Popkova, W.L. Nguyen, et al., J. Phys.: Conf. Ser., 1210, 012114 (2019) doi: 10.1088/1742-6596/1210/1/012114

15. H. Gao, M.K. Stenstrom, Water Res., 143, 445-56 (2018) doi: 10.1016/j.watres.2018.06.067

16. T.V. Le, T. Imai, et al., Chem. Eng. Sci., 94, 1-6 (2013) doi: 10.1016/j.ces.2013.02.044

17. N.A. Merentsov, V.A. Balashov, et al., IOP Conf. Ser.: Earth Environ. Sci., 224, 012041 (2019) doi: 10.1088/1755-1315/224/1/012041

18. A.B. Golovanchikov, V.A. Balashov, and N.A. Merentsov, Chem. Petrol. Eng., 53, 103 (2017) doi: 10.1007/s10556-017-0285-y

19. N.A. Merentsov, A.B. Golovanchikov, et al., J. Phys.: Conf. Ser., 1399, 055028 (2019) doi: 10.1088/1742-6596/1399/5/055028

20. T.N. Hunter, J. Peakall, et al., Chem. Eng. Sci., 218, 115560 (2020) doi: 10.1016/j.ces.2020.115560 